Submitted to Meta-Psychology. Participate in open peer review by commenting through hypothes.is directly on this preprint. The full editorial process of all articles under review at Meta-Psychology can be found following this link:

\title{
https://tinyurl.com/mp-submissions
}

You will find this preprint by searching for the first author's name.

\section{Writing a Psychological Blitz Literature Review with Nothing but a Browser}

\author{
Bogdan $\operatorname{Cocoş}^{1}$ \\ ${ }^{1}$ Department of Psychology, University of Bucharest
}

Author Note

Correspondence regarding this article should be addressed to Bogdan Cocoş, 90 Panduri Road, Sector 5, 050663, Bucharest, Romania. E-mail: bogdan.dm.cocos@gmail.com https://orcid.org/0000-0003-4098-7551 


\begin{abstract}
The ways so far of writing literature reviews represent valid, but not sufficient, landmarks, connected to the current technological context. In this sense, this article proposes a research method called blitz literature review, as a way to quickly, transparently, and repeatably consult key references in a particular area of interest, seen as a network composed of elements that are indispensable to such a process. The tutorial consists of six steps explained in detail, easy to follow and reproduce, accompanied by publicly available supplementary material. Finally, the possible implications of this research method are discussed, being brought to the fore a general recommendation regarding the optimization of the citizens' involvement in the efforts and approaches of open scientific research.
\end{abstract}

Keywords: blitz literature review, open access, open science, research methods 


\section{Writing a Psychological Blitz Literature Review with Nothing but a Browser}

\section{Context}

The term "blitz literature review" refers to an adaptation of the concept of literature review. The latter is defined as an effort to systematize information in a particular field, or on a certain topic of interest, an effort based on the review of knowledge so far (Snyder, 2019). We can understand, from the previously stipulated, that a systematic review of the literature is a sinuous process. Thus, the concept of blitz literature review emerges. Like the flash of a camera, this literature review can be intense and short-lived. The author captures the most relevant articles to date, in a quick but comprehensive manner.

\section{Reason}

Any reader may wonder where the need for such a piece of knowledge comes from. Of course, given that a keyword like "COVID-19" records, by the end of March 2021 alone, over 50,000 results, in Google Scholar, we can appeal to the pandemic, on motivation.

On a more serious note, this article appears for an almost obvious reason: both people in academia and those in the industry are required to compose projects, research, reports, or communiqués on certain topics of interest. If, to these steps, numerous articles are added, seemingly, "overnight”, problems may arise related to incomplete products or services. However, such incomplete products or services may have the potential to distort decisions with more or less increased risks.

\section{Generating Incompleteness}

One of the central reasons for the appearance of incomplete products or services is the very lack of transparency and reproducibility of scientific research. Examples can be facilitated by a case study: A consultant provides his psychological services in a company. 
The issue is related to a percentage of $40 \%$ of the company, characterized by employees who feel dissatisfied with their job, thus expressing their intentions to leave the organization. The consultant in question comes up with the idea of a service for exploring the unconscious and employees' relationships with their parents, in early childhood, as a way of solving this problem. In the package, the consultant also brings scientific articles that attest to the positive effects on his intervention. In short, the employee resolves certain unconscious conflicts, from early childhood, and will suddenly give up the intention to leave the organization. Even more, the performance of that employee will increase by $500 \%$, because "that's what science says!". Here, however, comes the dilemma: when we look for the consultant's articles, to check something that "sounds too good to be true", we either can't find them or we find them, but they are not publicly available. What are we going to do?

The previous example is a handy one, intentionally exaggerated, to capture the risks to which people are exposed, when it comes to questionable science. We can see that there is a need to accelerate transparent, verifiable, and repeatable scientific research - a science that everyone can access and verify or even carry out without considerable effort or waste of resources; a science that seeks authenticity, to the detriment of abracadabra results; objective and comprehensible science communication, to the detriment of the jargonesque gate-keeping (see Langin, 2021). Such an alternative is called open science.

\section{Rescue in Such Crisis Situations: Open Science}

In this sense, we can say that the acceleration of scientific research is the step we have to take. It is important to note that open science is an umbrella concept. In other words, no consensus has been reached at present on its definition; but rather to the classification of its elements. Before attempting to reproduce a definition of the field of open science itself, which has been recently postulated, the following discussion is needed. 
Therefore, favoring and encouraging open science can present it as a valid, intensively sought-after catalyst for scientific research (Kadakia et al., 2021). It is important to note that the dimension of open science does not stop at open access to articles or research data, but also at additional elements such as reproducibility, evaluation, policies, and tools.

Indeed, open access is the most popular dimension of open science (Researchers, research office, and libraries, 2020), but we cannot reduce the importance or validity of other dimensions to its detriment. Like any umbrella term, open science should not receive restrictive definitions, but rather should be seen as a set of elements, as a political and legal framework, focusing on the notions of validity, visibility, and reusability, specific to freely distributed materials (Gomez-Diaz \& Recio, 2020). Additionally, the freedom associated with distributed materials is not synonymous with gratuitousness and should not be understood as such (see the distinction between "gratis" and "libre"; Suber, 2008).

\section{Getting to the Point: Where Do We Go Now?}

Now that we have an introduction to the case, we can get to the central discussion of this article, namely: How can we write a blitz literature review, using only our browsers?

Before starting to argue, it should be noted that this information is a perfectly legal recommendation and, in theory, with zero costs involved. However, the products and services that can be obtained, using this summary of recommendations, have nothing to lose, in terms of real and perceived quality of work. Just because a work process involves automation and reduced effort, it does not mean that the work loses its overall quality. We must pay attention, however, to the quality and performance standards we are aiming for. These automated dimensions do not eliminate the responsibility to check our products or services offered, but only facilitate our steps. 
To visualize the actual delivery of a product, this article will be presented in the form of a tutorial, referring to the concept of competencies, described by Hoffmann (1999).

\section{Step One: Choosing the Ecosystem}

Before the actual realization of the project, we will need to ask the following question: “What are we working with?”. For a blitz literature review, there is no need for data analysis, therefore we will not discuss such applications.

It is metaphorically called an "ecosystem" that suite of applications used to carry out a project. If, for example, more than two Google applications are used, we may consider working in the Google ecosystem. If more than two Microsoft applications are used, we may consider working in the Microsoft ecosystem. The examples can be numerous and diverse. However, most of the time, we will refer to a mixed ecosystem, combining elements from multiple ecosystems, depending on utility, functionality, respectively other resources (e.g., temporal, financial).

When it comes to items like a browser or a search engine, the vast majority uses Google services. According to StatCounter, 63,59\% of the world's population uses Google Chrome, Google's browser (https://gs.statcounter.com/browser-market-share); and 92,05\% of the world's population uses the Google search engine. (https://gs.statcounter.com/search-engine-market-share).

What about people who want to use alternatives to these products and services? Especially for people interested in online privacy, this market share of the Google ecosystem may seem daunting. However, in short, any Chromium-based browser (i.e., Chromium is the open-source project of Google) (e.g., Google Chrome, Microsoft Edge, Opera, Brave, Vivaldi, etc.); or any Gecko-based browser (i.e., Gecko is the open-source project of Mozilla) (e.g., Firefox, Waterfox, K-Meleon, LibreWolf, etc.) proves to be capable of undertaking the 
work necessary to carry out such projects. In terms of search engines, there are alternatives such as Bing, DuckDuckGo, Qwant, Ecosia, Startpage, some of which even offer the results offered by Google, but with more robust online privacy policies.

After selecting a browser, you will need to select a search engine; but not just any search engine, but one geared toward academic results. Such search engines, which offer specific capabilities of open science, are Google Scholar, Microsoft Academic, Internet Archive Scholar, fatcat! wiki and more. Of course, there may be opponents of these platforms, especially in the case of Google Scholar (see Bastian, 2019), but, for a blitz literature review, we can consider such platforms as sufficient.

Regarding the creation of this article, Brave browser version 1.22.70 was used in the Pop!_OS 20.04 LTS distribution of Linux (i.e., operating system), the academic search engines being the four mentioned above.

\section{Step Two: Identifying the Central Node and Building the Knowledge Network}

Next, we will need to discuss the network-type route of a literature review process. Any network, in a minimal sense, is composed of two elements: nodes (i.e., variables of interest) and edges (i.e., the relationships between those variables) (Hevey, 2018). Web applications such as Connected Papers, ResearchRabbit, Open Knowledge Maps, Inciteful (https://inciteful.xyz), or many others will be used to generate networks of scientific articles based on a variable of interest. Regarding the creation of this article, the Connected Papers web application was used.

In this article, the central node is represented by the construct of competency. Thus, a first step is to write the term "competency" in one of our academic search engines. The first result is an article identified as the most relevant, based on the number of citations, namely that of Hoffmann (1999). The next step is to select a permanent link or identification code, 
such as the article-specific DOI code, followed by inserting the selection into one of the network generation engines. Connected Papers generated, for Hoffmann's (1999) article, the network that can be seen in Figure 1.

\section{Figure 1}

The network of scientific articles generated by Connected Papers

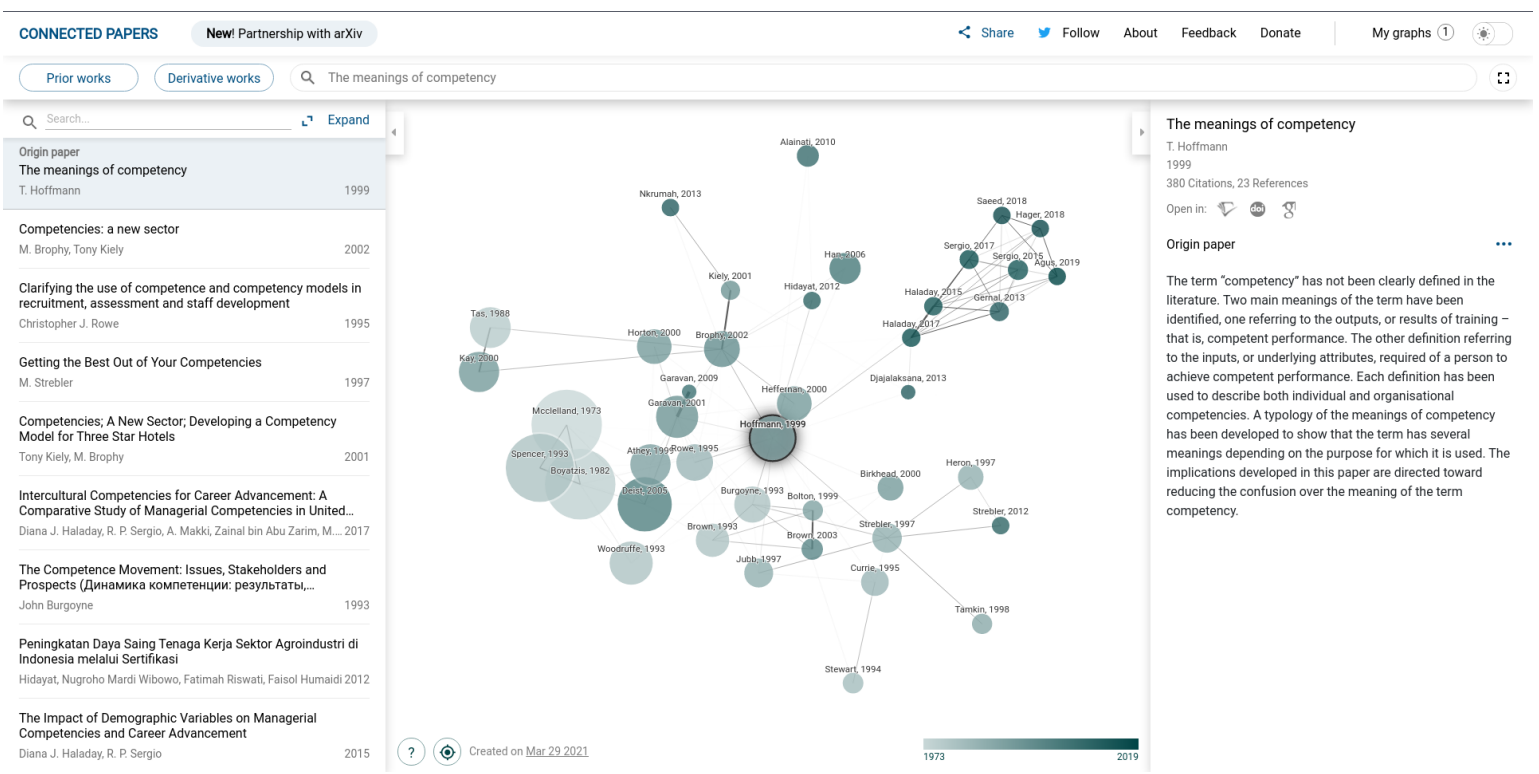

Based on the network of scientific articles, articles of interest for the blitz literature review can be easily identified.

\section{Step Three: Identifying Key References, or Citation Chasing}

Now that we have the network of articles of interest, we are particularly interested in which articles the author of our central node cited, respectively which articles cite this node, depending on the years. These facts will allow us to connect to the entire history of the central node, the process of "tracking the steps" of an author being called "citation chasing" (University of Illinois Library, 2021).

To streamline the citation chasing process, the Shiny application citationchaser (Haddaway et al., 2021), available at https://estech.shinyapps.io/citationchaser/, will be used. 
To obtain the necessary data, in the Article input section, enter the DOI code of the selected article. This action will generate a list of bibliographic references contained in the original article of the author, a list of bibliographic references that cite the article selected by us, respectively a network of these. Bibliographic reference lists can be saved in the ris format, containing the citation metadata of the articles and the abstract of each, where applicable. For the example in this article, the bibliographic reference lists in the ris format can be found in the OSF link specific to additional materials.

\section{Step Four: Legal Access to Knowledge}

Although the academic search engines mentioned earlier provide links to articles, in open access regime, some articles may remain impossible to access. In this regard, browser extensions, such as Unpaywall and Open Access Button, can be used to legally access articles of interest. These extensions work on both Chromium-based and Gecko-based browsers, without the need for skills or competencies specific to tech-savvy people to use them.

If an article still cannot be accessed, it is recommended to contact the authors of the text directly, with the request to distribute a copy, for its use for research purposes or profit. In contacting the authors, transparency and respect are recommended (i.e., the reason for the request and the implications of using the article will be specified).

To access the scientific articles used in this paper, the open access capabilities of the academic search engines and the Unpaywall extension were used. It is important to note that the focal article, that of Hoffmann (1999), is not offered in a legal open access regime.

\section{Fifth Step: Preparing the Project}

Returning to the discussion related to the ecosystem, in this step of elaboration of the project, a text editor and a word processor are needed. Contrary to expectations, the most used office suite is not the one offered by Microsoft, but the alternative offered by Google, 
with a percentage of $58.82 \%$ of users, globally, according to Datanyze (https://www.datanyze.com/market-share/office-suites--370). We can argue this percentage in terms of the fact that the alternative offered by Google is completely gratis and its capabilities are vast, thanks to the add-ons.

Of course, the preparation of the project can take place with the help of any office suite, from proprietary software (e.g., Microsoft Office, G Suite, WPS Office, etc.) to FOSS (i.e., free \& open-source software) (e.g., LibreOffice, Apache OpenOffice, ONLYOFFICE, etc.). However, the use of an office suite that would require downloading and installing software would not fall within the discussion of this article.

Among the examples of online office suites, for working strictly in a browser, we can mention, in addition to those aforementioned, Zoho Office Suite, iWork for iCloud, Collabora Online, and Feng Office. These may be bundled with incompatibilities with certain document-specific types of formats, so careful research and selection of the office suite used are recommended.

As in any project, the correct citation of sources is essential. In this sense, platforms such as Google Scholar offer the possibility to cite the materials used, but, depending on the writing styles chosen or imposed, these citations may be erroneous or incomplete (Figure 2). Thus, additional platforms such as ZoteroBib, Citefast, Citation Machine, EasyBib, Scribbr Citation Generators, and many others are recommended for generating bibliographic references according to the chosen or imposed writing style.

\section{Figure 2}

Citation example provided by Google Scholar 


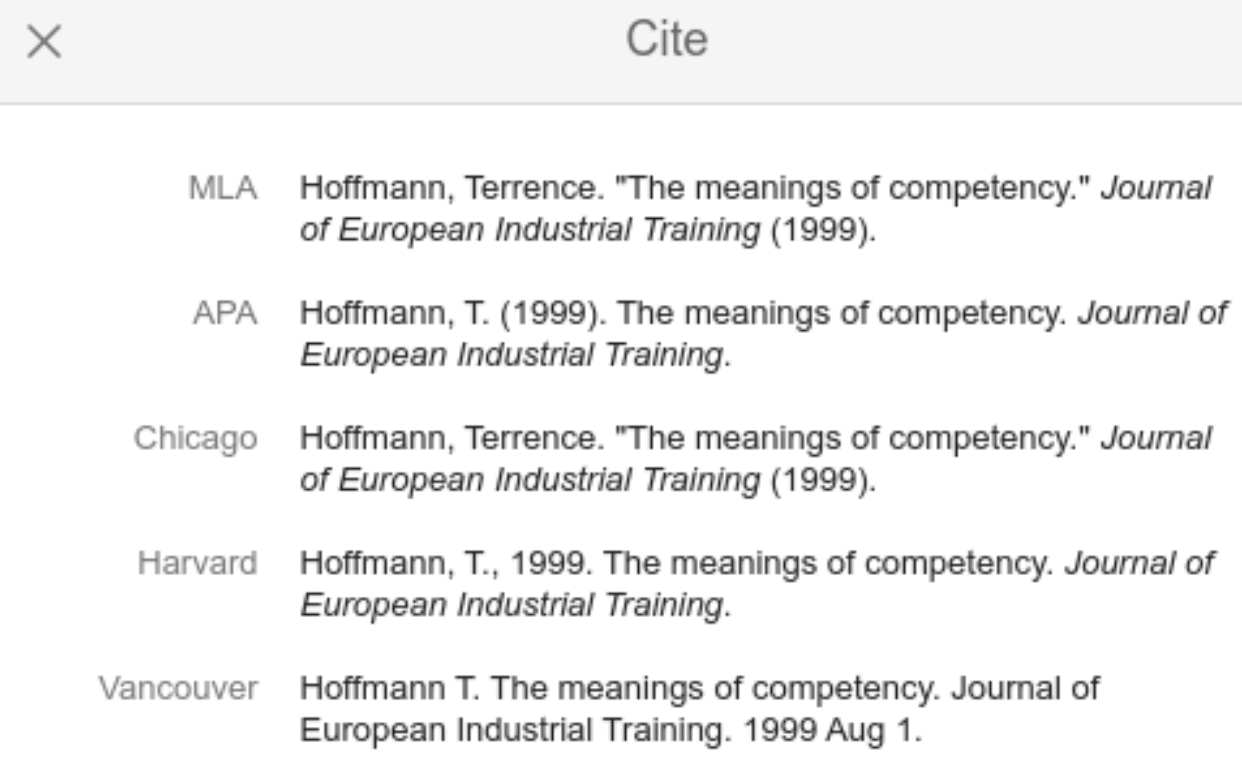

MLA Hoffmann, Terrence. "The meanings of competency." Journal of European Industrial Training (1999).

APA Hoffmann, T. (1999). The meanings of competency. Journal of European Industrial Training.

Chicago Hoffmann, Terrence. "The meanings of competency." Journal of European Industrial Training (1999).

Harvard Hoffmann, T., 1999. The meanings of competency. Journal of European Industrial Training.

Vancouver Hoffmann T. The meanings of competency. Journal of European Industrial Training. 1999 Aug 1.

\section{BibTeX EndNote RefMan RefWorks}

Additionally, in any article, citations within the text must be found in the bibliographic references, without exception. To automate such a difficult process, in the case of numerous citations and bibliographic references, it is recommended to use the Recite web application (https://reciteworks.com/). It has a minimalist interface (Figure 3), being optimized for APA and Harvard writing styles.

\section{Figure 3}

Overview of the Recite platform 


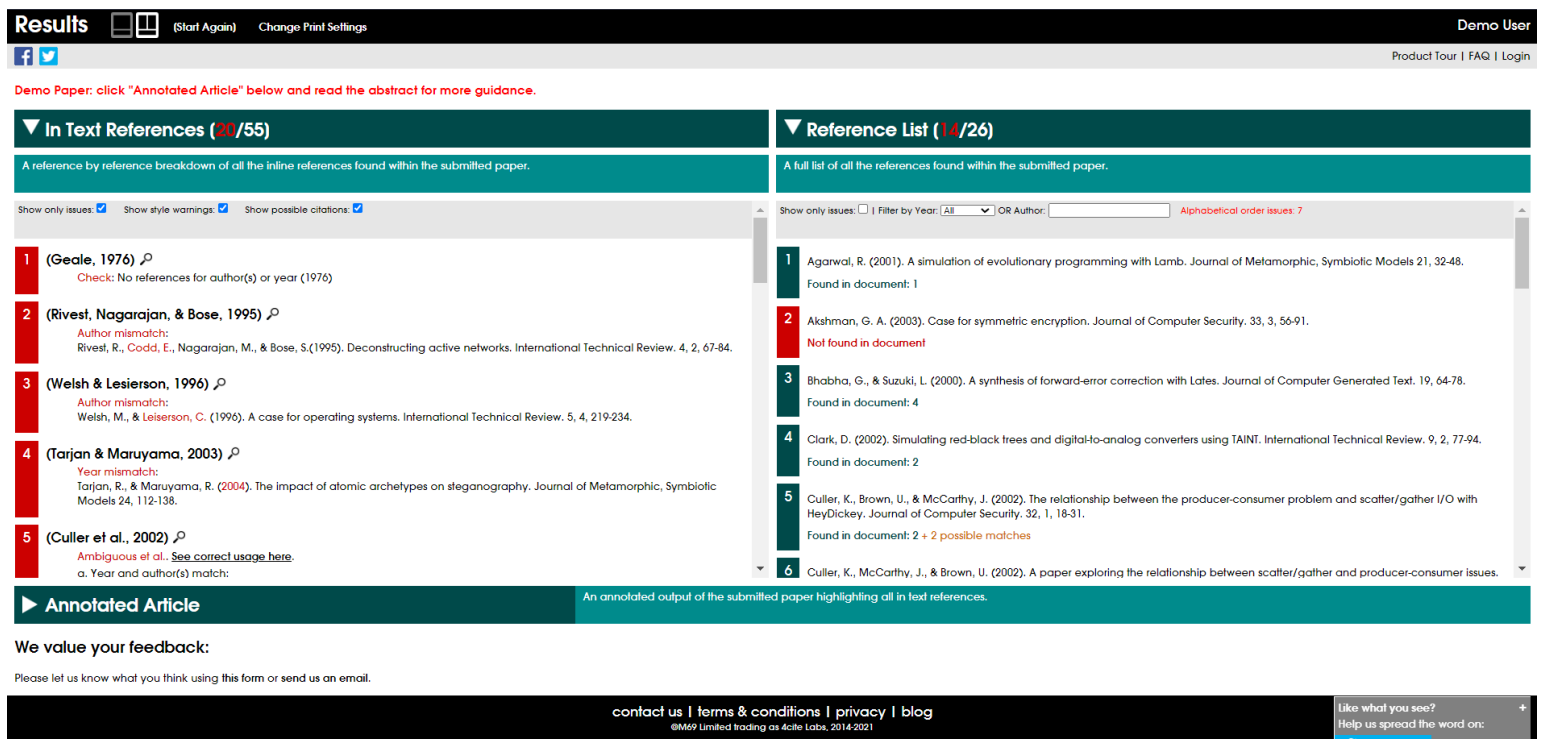

Another important chapter is the one related to reading or browsing the selected materials, respectively to verifying their veracity. There may be a rational need to quickly go through an article of considerable size and to see if it continues to be a valid point of reference in the literature. Online services such as Scholarcy and Paper Digest, for summarizing articles of interest, respectively scite (https://scite.ai/), for verifying the authenticity (i.e., how supported the article is by other authors or how much it is debated in the literature) can facilitate the steps of synthesis and optimal communication of focal evidence. For example, the visual report generated by scite will be presented, this platform generating a network that is similar to the one generated by Connected Papers (Figure 4).

\section{Figure 4}

The visual report generated by scite 


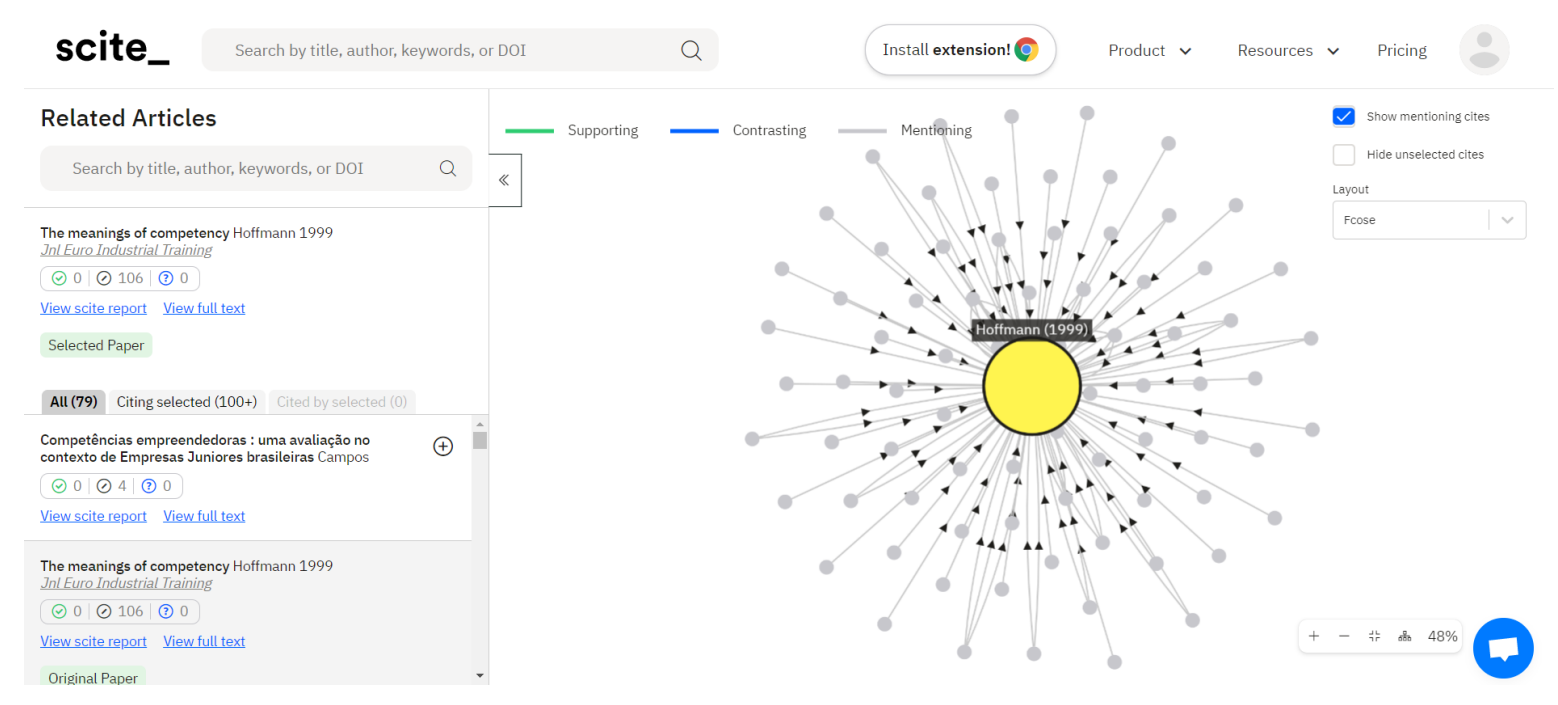

Google Docs, from the office suite provided by Google, was used to effectively write this article, along with the Sorted Paragraphs add-on (https://workspace.google.com/marketplace/app/sorted paragraphs/658758119473), for the alphabetical ordering of bibliographic references. ZoteroBib, with the option of the American Psychological Association 7th edition, regarding the writing style, was used to create and cite the bibliographic references. The network for verifying the veracity of Hoffmann's (1999) article was created using scite. Given that Hoffmann's (1999) article is not offered in a legal open access regime, a summary of it with the help of Scholarcy could not be put into practice. However, a related article, that of Bartram (2005), was selected for exemplification (Figure 5), which is available in legal open access regime, through ResearchGate.

\section{Figure 5}

Scholarcy overview 


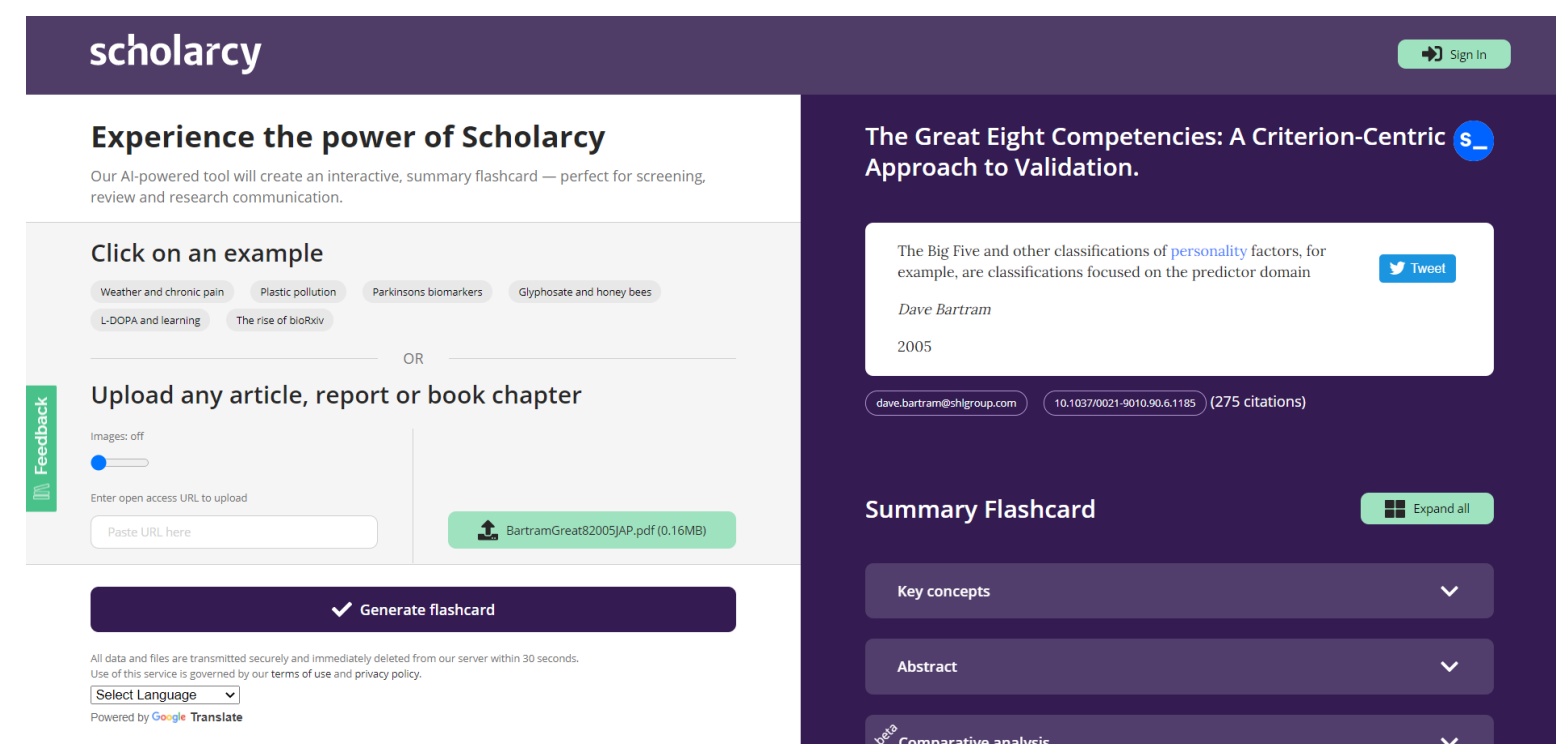

\section{Sixth and Last Step: Communicating Results and Effective Product Delivery}

This last step answers the question "How can I communicate the obtained results?".

Since the late 1990 s and early 2000 s, the communication of results in the business

environment was facilitated by the use of visual aids built using presentation software such as Microsoft PowerPoint (McCannon \& Morse, 1999). Today, we can see greater openness to gratis alternatives with extensive capabilities, such as Google Slides, Canva, Prezi, Microsoft PowerPoint Online, and Microsoft Sway.

Now that we have the visual support to present and communicate our material, we will need to find a way for interested people to access that material. Such ways can come either by distributing links to the materials themselves or by creating permanent links for them. Among the platforms that can generate permanent links and DOI identification codes, we mention Open Science Framework (OSF), figshare, Zenodo, and F1000Research.

Usually, when it comes to delivering a product, there are elements of notions such as intellectual property and copyright. For a clearer understanding and application of distributed material licenses, it is recommended to consult the Creative Commons website (https://creativecommons.org/). 


\section{Summary}

Therefore, the previously developed steps will be captured in specific summaries, as follows.

Ecosystem choice refers to the actual environment in which the work takes place. From the operating system and browser to the search engines used, all of these elements make up the first step in a blitz literature review.

The identification of the central node and the construction of the knowledge network refers to the identification of a starting point in the blitz literature review. This starting point comes in the form of a variable of interest (i.e., which can be a concept, an article, a book, etc.), around which there currently is knowledge. Of course, knowledge is interconnected, being easy to understand in terms of a network of knowledge or articles.

The identification of key references can be understood in terms of "following the steps" that made it possible to generate the variable of interest. The key references speak about the knowledge up to the variable, respectively about the knowledge subsequent to the highlighting of the variable. A process of identifying the key references is needed to be able to connect to the retrospective, present, and prospective knowledge of the variable of interest.

Legally accessing knowledge is the next step in identifying key references. These references have been identified and will then be read, downloaded, used, and reused, distributed, or redistributed following a pre-established action plan. To this end, there are legal ways to access knowledge, which can contribute to the maximum preparation of the project.

The elaboration of the project is the step of effective writing or composing the material. In this step, we can "put on paper" the results of previous efforts, and they will be further disseminated and delivered. 
Communicating the results and the actual delivery of the product is the last step in the list described, referring to how the results put on paper, in the previous step, acquire forms and meaning that can be applied, depending on different subjective or objective needs. At the same time, a crucial aspect of this step is how the material is delivered and how it can be used or reused, on a case-by-case basis. To this issue, an author can find the answers sought in the licenses on copyright and intellectual property, specific to his or her work.

\section{Conclusions and Discussions}

It is hoped that such an article will optimize future efforts and research of authors interested in the psychological literature. To date, we cannot say with certainty that there have been systematic and systematized initiatives to guide the efforts behind rapid reviews of the literature in the context of open science.

Indeed, there are current methodologies, specific to rapid reviews of the literature, but they appeal to certain constraints related to research resources (e.g., a period of 1-6 months, exclusion of manual searches or non-indexed literature, criteria for exclusion based on age and language; VCU Libraries Research Guides, 2018). Besides, the methodologies behind these rapid literature reviews do not take open science into account as a primary factor.

Of course, there may be limitations to such an approach. In this regard, the article has been published under the Attribution 4.0 International license (CC BY 4.0), so that anyone who sees the opportunity to modify this manuscript is free to remove or add information, depending on the expertise and needs of customers or stakeholders.

In conclusion, such an approach has the potential to open science and familiarize both researchers and anyone who wants to access the information in question, with best practices on scientific conduct. The recommendation that can be deduced from this article is the 
involvement of all citizens in the processes behind scientific research, respectively the popularization of science among citizens, to optimize current theories and practices.

\section{Conflict of Interest and Funding}

The author does not report conflicts of interest or funding.

\section{Author Contributions}

The entire manuscript was composed and revised by the author. 


\section{References}

Bartram, D. (2005). The great eight competencies: A criterion-centric approach to validation. Journal of Applied Psychology, 90(6), 1185-1203. https://doi.org/10.1037/0021-9010.90.6.1185

Bastian, H. (2019, September 27). Google scholar risks and alternatives. Absolutely Maybe. https://absolutelymaybe.plos.org/2019/09/27/google-scholar-risks-and-alternatives/

Gomez-Diaz, T., \& Recio, T. (2020). A policy and legal Open Science framework: A proposal. ArXiv:2010.04508 [Cs]. $\underline{\text { http://arxiv.org/abs/2010.04508 }}$

Haddaway, N. R., Grainger, M. J., Gray, C. T. (2021) citationchaser: An R package and Shiny app for forward and backward citations chasing in academic searching. https://doi.org/10.5281/zenodo.4543513

Hevey, D. (2018). Network analysis: A brief overview and tutorial. Health Psychology and Behavioral Medicine, 6(1), 301-328. https://doi.org/10.1080/21642850.2018.1521283

Hoffmann, T. (1999). The meanings of competency. Journal of European Industrial Training, 23(6), 275-286. https://doi.org/10.1108/03090599910284650

Kadakia, K. T., Beckman, A. L., Ross, J. S., \& Krumholz, H. M. (2021). Leveraging open science to accelerate research. New England Journal of Medicine, Article NEJMp2034518. https://doi.org/10.1056/NEJMp2034518

Langin, K. (2021, April 6). Want other scientists to cite you? Drop the jargon. Science. https://doi.org/10.1126/science.caredit.abi8836

McCannon, M., \& Morse, G. E. (1999). Using multimedia visual aids in presentations: The demise of the transparency has been greatly exaggerated. TechTrends, 43(6), 29-31. https://doi.org/10.1007/BF02818219 
Snyder, H. (2019). Literature review as a research methodology: An overview and guidelines. Journal of Business Research, 104, 333-339.

https://doi.org/10.1016/j.jbusres.2019.07.039

Suber, P. (2008). Gratis and libre open access. SPARC Open Access Newsletter.

https://dash.harvard.edu/handle/1/4322580

The growing popularity of open access: Researchers, research office, and libraries. (2020, October 6). Ex Libris.

https://exlibrisgroup.com/blog/popularity-open-access-researchers-research-office-libr $\underline{\operatorname{aries} /}$

University of Illinois Library. (2021, February 22). Libguides: Advanced research at the library: citation chasing. Retrieved March 29, 2021, from https://guides.library.illinois.edu/c.php?g=563215\&p=3877582

VCU Libraries Research Guides. (2018, September 11). Rapid review protocol. Retrieved March 30, 2021, from https://guides.library.vcu.edu/rapidreview 


\section{Supplemental Material}

All additional files can be accessed on OSF: $\underline{\text { https://osf.io/tx6sh/ }}$ 